\title{
TEDDEEC: Threshold Enhanced Developed Distributed Energy-Efficient Clustering Protocol for Heterogeneous Wireless Sensor Networks
}

Aditi Sharma, Harjit Singh

Student of Electronics and Communication, Guru Nanak Dev University, Punjab aditi.sharma.btech.892700@gmail.com

Faculty of Electronics and Communication, Guru Nanak Dev University, Punjab

hs_kahlona@yahoo.com

\begin{abstract}
In wireless sensor networks, hundreds or thousands of sensor networks are deployed in a field to sense data (like temperature, light, pressure, sound etc.) and then they transmit it to sink nodes or base station via a radio transmitter. At one side where these networks have numerous applications, they suffer from a major disadvantage on the other side. These sensor nodes are power constrained and hence they have limited lifetime. Once deployed, these nodes cannot be recharged, therefore researchers are developing protocols to enhance network lifetime. Protocols had been modified one after the other so as to save energy during their every transmission. Clustering technique was used and it was found that it is more optimistic way to save energy. In this paper, we propose a new clustering protocol: Threshold Enhanced Developed and Distributed energy-Efficient Clustering Protocol (TEDDEEC) for heterogeneous wireless sensor networks. In this technique, a modified value of threshold is presented on which a node will decide whether to become $\mathrm{CH}$ or not. Simulation results show that this protocol outperforms as compared to its conventional counterparts.
\end{abstract}

\section{Indexing terms/Keywords}

TEDDEEC; heterogeneous WSNs; clustering; threshold; network lifetime

\section{Academic Discipline And Sub-Disciplines}

Engineering, Electronics and Communication Engineering, Specialization in Communication

\section{SUBJECT CLASSIFICATION}

Communication

\section{TYPE (METHOD/APPROACH)}

Analysis and Modeling

\section{Council for Innovative Research}

\section{Peer Review Research Publishing System}

\section{Journal: INTERNATIONAL JOURNAL OF COMPUTERS \& TECHNOLOGY}

Vol.14, No.6

www.ijctonline.com, editorijctonline@gmail.com 


\section{INTRODUCTION}

Wireless sensor network technology is the key technology for future. Sensor nodes are the most important component in wireless sensor network. These sensor nodes have four subsystems viz. power subsystem, processor, communication subsystem and sensor subsystem. These nodes are power constrained i.e. they have limited lifetime [1]. So, various routing protocols were developed to reduce the energy consumption by applying clustering technique. This technique includes formation of clusters in which cluster member nodes send the sensed data to their respective cluster heads and these $\mathrm{CHs}$ transmit it to base station through a radio channel after data aggregation [2]. Clustering is done in two types of networks: Homogeneous and Heterogeneous networks. Both the networks differ from each other in the sense of energy level of $\mathrm{CHs}$. In homogeneous all nodes have same energy level. Whereas, in heterogeneous networks nodes have different initial energies. Few examples for homogeneous network protocols are LEACH (Low Energy Efficient Adaptive Clustering Hierarchy) [3], HEED (Hybrid Energy Efficient Distributed Clustering) [4], PEGASIS (Power Efficient Gathering in Sensor Information Systems) [5]. These protocols fail to perform well in heterogeneous network because in these protocols nodes are not treated with respect to energy. In heterogeneous WSNs, nodes are differentiated with respect to their residual energy. SEP (Stable Election Protocol) [6], DEEC (Distributed Energy-Efficient Clustering) [7], DDEEC (Developed Distributed Energy-Efficient Clustering) [8], EDEEC (Enhanced Distributed Energy-Efficient Clustering) [9], EDDEEC (Enhanced Developed Distributed Energy-Efficient Clustering) [10] are few examples of heterogeneous WSN protocols. In Section II, TEDDEEC protocol has been described. In section III, simulations and results are shown. Finally in section IV, work is concluded.

\section{TEDDEEC PROTOCOL}

TEDDEEC implements the same strategy for estimating the energy in the network as proposed in EDDEEC [10]. The average energy of $r$ th round is given as:

$$
\overline{\mathrm{E}}(r)=\frac{1}{N} E_{\text {total }}\left(1-\frac{r}{R}\right)
$$

$R$ be the total rounds during network lifetime and can be estimated from [10] as:

$R=\frac{E_{\text {total }}}{E_{\text {round }}}$

$E_{\text {round }}$ is the energy dissipated in a network during single round and can be calculated as:

$E_{\text {round }}=L\left(2 N E_{\text {elee }}+N E_{D A}+k \varepsilon_{\text {mp }} d_{\text {toBs }}^{4}+N \varepsilon_{f s} d_{\text {toCH }}^{2}\right)$

where $\mathrm{k}$ is number of clusters dtoBs is the average distance between cluster head and the base station and $\mathrm{d}$ to $\mathrm{CH}$ is the average distance between the cluster members and the cluster head.

Now $\mathrm{d}$ toBS and $\mathrm{d}$ to $\mathrm{CH}$ can be calculated as:

$d_{\text {tOCH }}=\frac{M}{\sqrt{2 \pi k}}, d_{\text {tOBS }}=0.765 \frac{M}{2}$

By calculating the derivative of $E_{\text {round }}$ with respect to $k$ to zero we get optimal number of clusters as:

$k_{\text {opt }}=\frac{\sqrt{N}}{\sqrt{2 \pi}} \sqrt{\frac{\varepsilon_{f s}}{e_{\text {pmp }}}} \frac{M}{d_{\text {toBs }}^{2}}$

Hence we can find the energy dissipated per round by substituting equations (4) \& (5) in (3).Due to the heterogeneity factors $R$ is taken as $1.5 \mathrm{R}$.

Traditionally, as per EDDEEC, cluster head algorithm is broken into rounds. At each round node decides whether to become a cluster head based on threshold calculated by the suggested percentage of cluster heads for the network and the number of times the node has been a cluster-head so far. This decision is made by the nodes by choosing the random number between 0 and 1 . If the number is less than a threshold $T(s)$ the node becomes a cluster-head for the current round. The threshold is set as:

$T(s)=\left\{\begin{array}{l}\frac{p_{\mathrm{i}}}{1-p_{\mathrm{i}}\left(\operatorname{rmod} \frac{1}{p_{\mathrm{i}}}\right)} \text { if } s_{\mathrm{i}} \in G \\ 0 \quad \text { otherwise }\end{array}\right.$

where $p, r$, and $G$ represent, respectively, the desired percentage of cluster-heads, the current round number, and the set of nodes that have not been cluster-heads in the last $1 / p$ rounds. Using this threshold, each node will be a cluster head, just once at some point within 1/p rounds. 
In TEDDEEC approach we have adjusted the value of the threshold, according to which a node decides to be a cluster head or not, based on ratio of residual energy and average energy of that round in respect to the optimum number of cluster heads. So that only nodes having a more energy becomes the cluster head.

The threshold Eq. (6) is set as:

$$
T(s)= \begin{cases}\frac{p_{\mathrm{i}}}{1-p_{\mathrm{i}}\left(\operatorname{rmod} \frac{1}{p_{\mathrm{i}}}\right)} * \frac{\text { Residual Energy of a node } * K_{\text {opt }}}{\text { Average energy of network }} s_{\mathrm{i}} \in G \\ 0 \quad \text { otherwise }\end{cases}
$$

The difference between DEEC, DDEEC, EDEEC, EDDEEC and TEDDEEC is generalized in Eq. 7, which defines threshold level for a node to decide whether to become $\mathrm{CH}$ for current round or not. Aim of this expression is to distribute energy consumption over network efficiently, increase stability period and lifetime of network.

The probabilities of normal, advanced and super nodes in case of two-level heterogeneity are same as that in case of EDDEEC [10]:

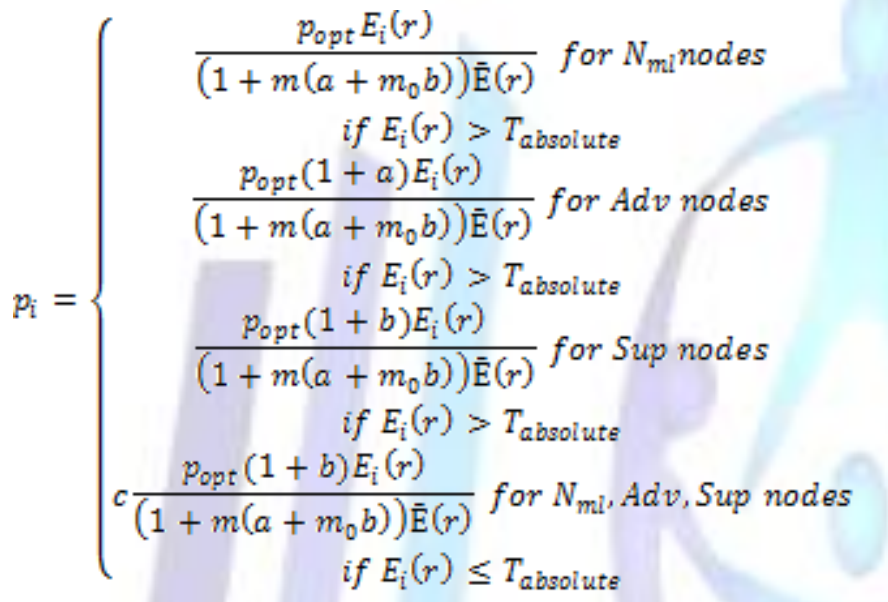

The value of absolute residual energy level, Tabsolute, is written as:

$$
T_{\text {absolute }}=z E_{0}
$$

where, $z \varepsilon(0,1)$. If $z=0$ then we have traditional EDEEC. In reality, advanced and super nodes may have not been a $\mathrm{CH}$ in rounds $r$, it is also probable that some of them become $\mathrm{CH}$ and same is the case with the normal nodes. So, exact value of $z$ is not sure. However, through numerous of simulations using random topologies, we try to estimate the closest value of $z$ by varying it for best result based on first dead node in the network and find best result for $z=0.7$. Therefore, Tabsolute $=$ (0.7)Eo.

Here, $\mathrm{c}$ is a real positive variable which control directly the clusters head number. On one hand, if $\mathrm{c}$ is higher, the number of cluster heads will increase. Then, the network scheme will be like a direct communication because all nodes will be a cluster head and transmit directly here information to the base station, in this case the network performances will increase. On the other hand, if $c=0$, the probability to be a cluster heads will be equal to zero for all nodes. So, they go to transmit directly their measurement to the base station, thus, they die quickly, that we want certainly to avoid. When simulated and analyzed, it was found that if $c$ is nearest to 0.02 we have more network performances [8].

\section{SIMULATIONS AND RESULTS}

In this section, we present simulation result for DEEC, DDEEC, EDEEC, EDDEEC and TEDDEEC for three-level heterogeneous WSNs using MATLAB. WSNs consist of $N=100$ nodes which are randomly placed in a field of dimension $100 \mathrm{~m} \times 100 \mathrm{~m}$. For simplicity, we consider all nodes are either fixed or micro-mobile and ignore energy loss due to signal collision and interference between signals of different nodes that are due to dynamic random channel conditions. In this scenario, we are considering BS is placed at center of network field.

The performance metrics used for evaluation of clustering protocols for heterogeneous WSNs are stability period, lifetime of the heterogeneous WSNs and data packets which are successfully sent to BS. In heterogeneous WSNs, we used radio parameters as mentioned in Table 1 below for different protocols deployed in WSNs and estimated performance for the case of three-level heterogeneous WSNs. 
Table 1. Experimental Setup

\begin{tabular}{|l|l|}
\hline Parameter & Value \\
\hline Area (x,y) & 100,100 \\
\hline Base station & 50,50 \\
\hline Nodes (n) & 100 \\
\hline Probability (p) & 0.1 \\
\hline Initial Energy (Eo) & 0.5 \\
\hline Transmitter_Energy & $50 \mathrm{~nJ} / \mathrm{bit}$ \\
\hline Receiver_Energy & $50 \mathrm{~nJ} / \mathrm{bit}$ \\
\hline Free Space (Amplifier) & $10 \mathrm{pJ} / \mathrm{bit} / \mathrm{sq} \cdot \mathrm{m}$. \\
\hline Multipath (Amplifier) & $0.0013 \mathrm{pJ} / \mathrm{bit} / \mathrm{sq} . \mathrm{m}$. \\
\hline $\begin{array}{l}\text { A (Energy Factor Between Normal } \\
\text { and Super Nodes) }\end{array}$ & 3.5 \\
\hline $\begin{array}{l}\text { B (Energy Factor Between Normal } \\
\text { and advance Nodes) }\end{array}$ & 2 \\
\hline Maximum Lifetime & \\
\hline Message size & 10000 \\
\hline M (Fraction of Advanced Nodes) & 0.8 \\
\hline X (Fraction of Super Nodes) & 0.6 \\
\hline Effective Data Aggregation & $5 \mathrm{~nJ} / \mathrm{bit} / \mathrm{signal}$ \\
\hline
\end{tabular}

We consider a network containing 20 normal nodes having E0 energy, 32 advanced nodes having 2.0 times greater energy as compare to normal nodes and 48 super nodes containing 3.5 times greater energy as compare to normal nodes. The parameters used for the simulation are given following:

- $\mathrm{m}=0.8$

- $\mathrm{mo}=0.6$

- $a=2.0$

- $b=3.5$

Figure 1 depicts number of alive nodes during lifetime of network. All nodes for DEEC, DDEEC, EDEEC, EDDEEC and TEDDEEC die at 5317, 5738, 8496, 8650 and 9993 rounds respectively.

Figure 2 depicts number of dead nodes during lifetime of network.

Figure 3 shows data packets sent to BS for DEEC, DDEEC, EDEEC, EDDEEC and TEDDEEC are 137823, 131591, 396746, 436792, 528188 respectively. Results show that TEDDEEC is most efficient among all protocols in terms of stability period, network life time and packets sent to BS even in case of network containing more super and advanced nodes as compared to the normal nodes.

Figure 4 depicts remaining energy for each protocol. With the same parameters and same initial energy, TEDDEEC has maximum total energy (or remaining energy) at every round with respect to other nodes. 


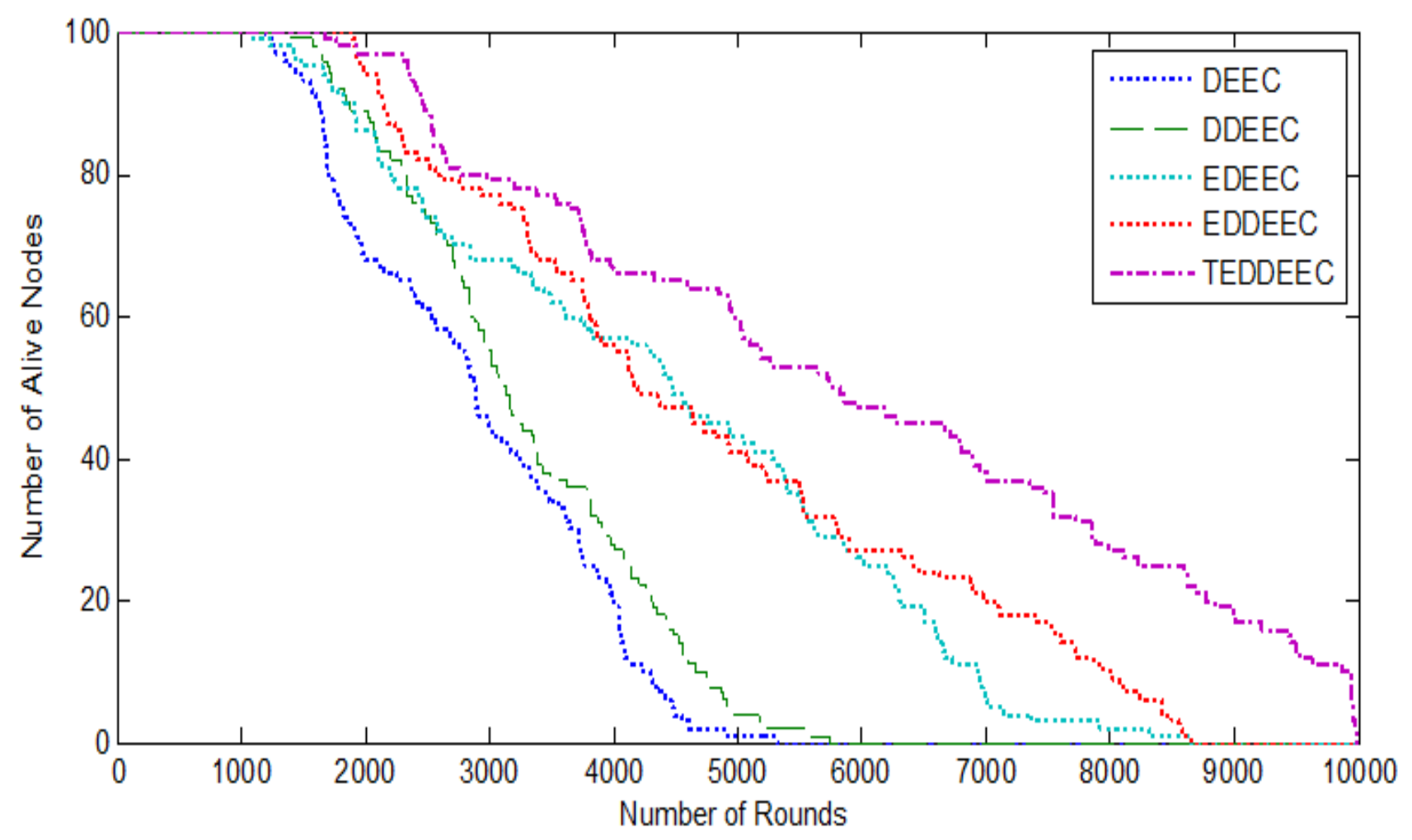

Figure 1 Alive nodes during network lifetime

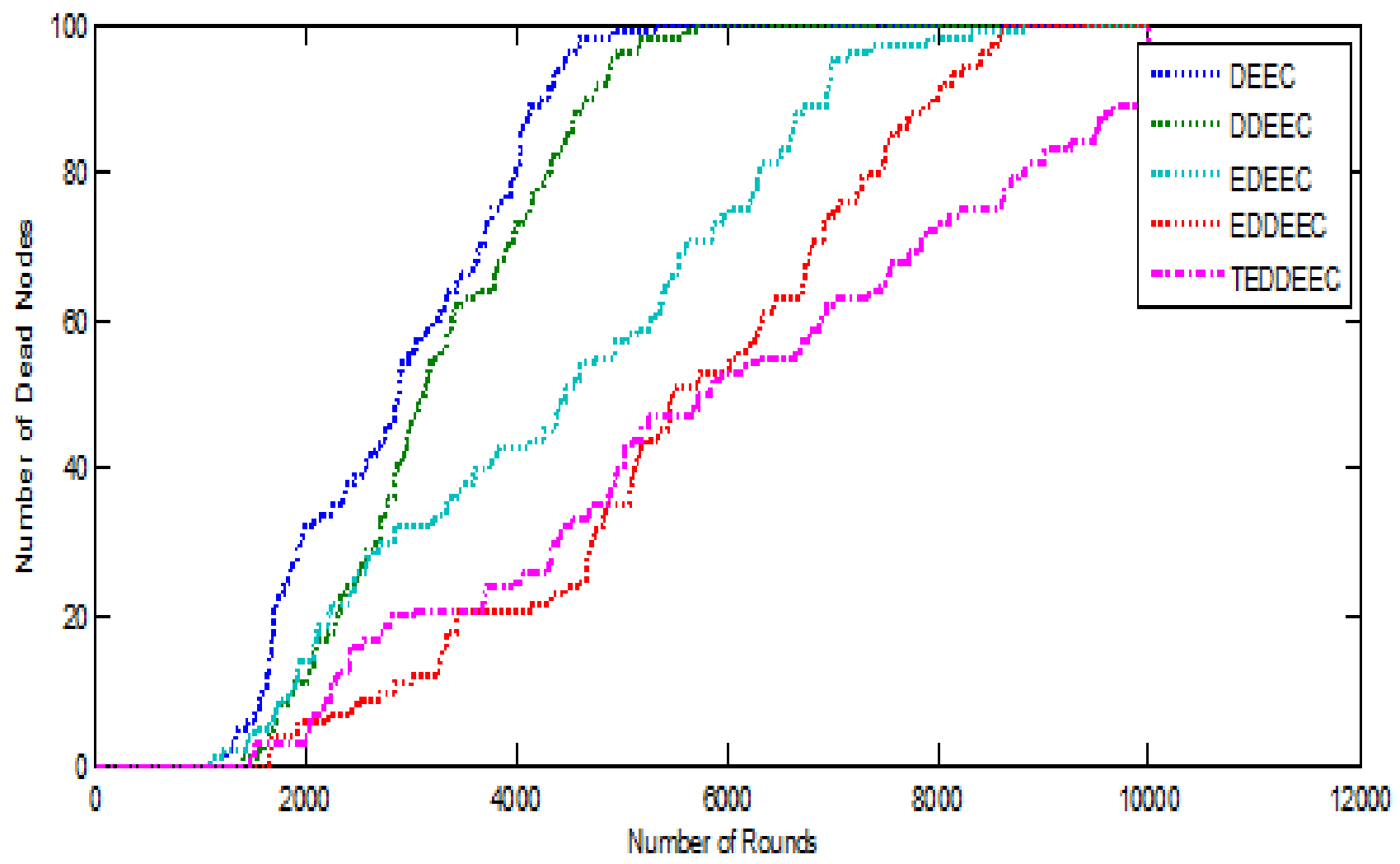

Figure 2 Number of dead nodes during network lifetime 


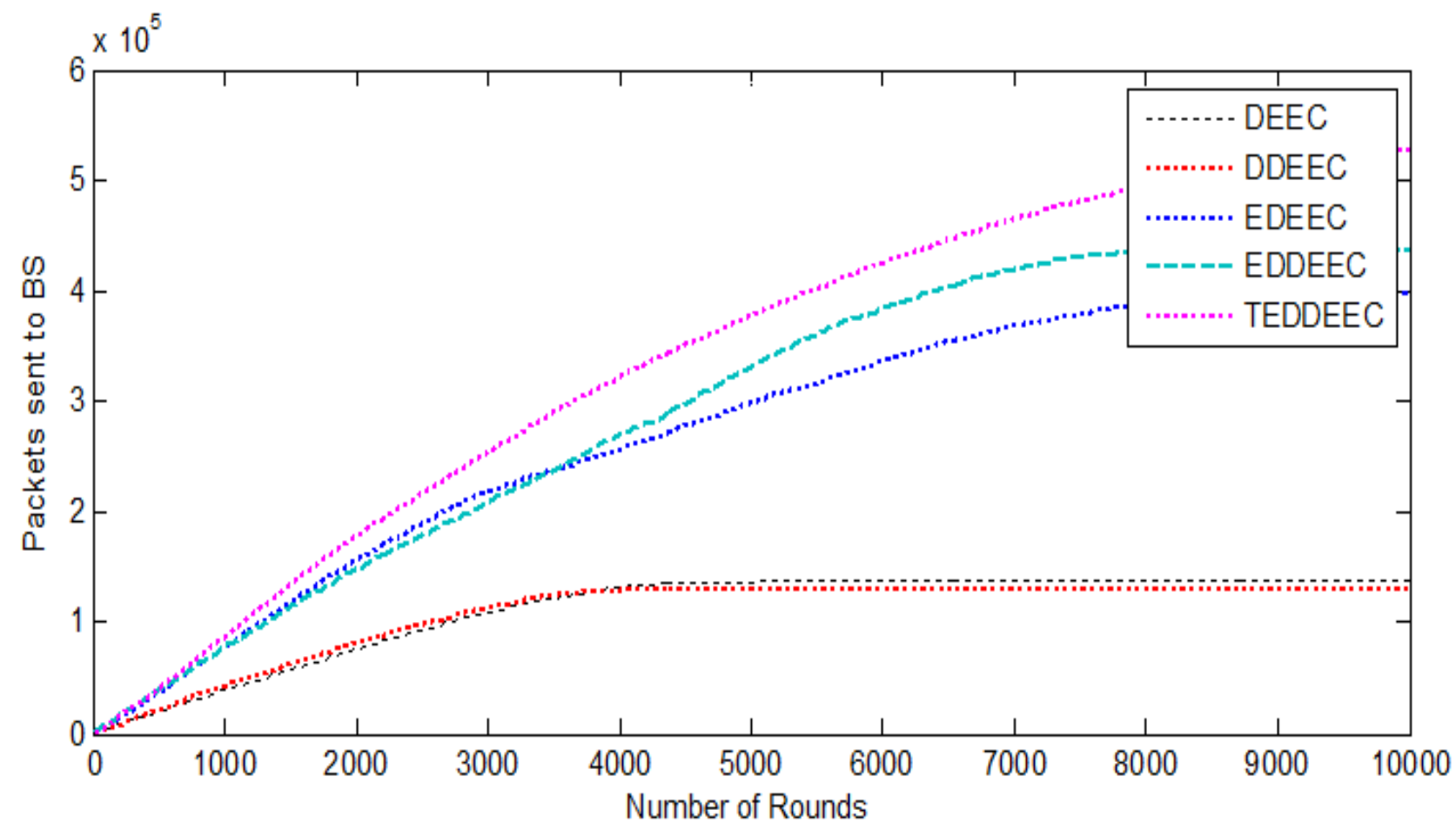

Figure 3 Packets send to base station

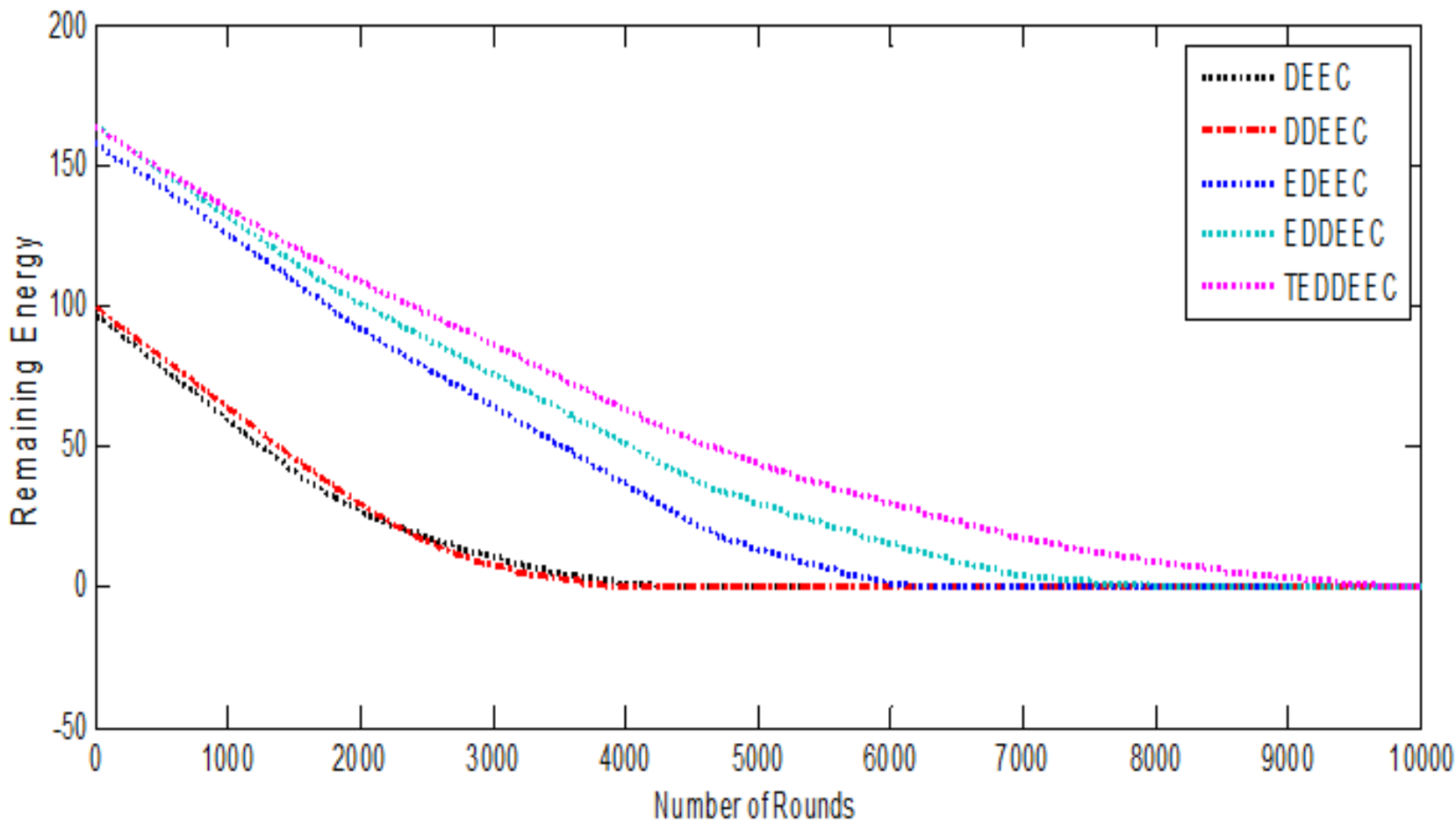

Figure 4 Remaining Energy

\section{CONCLUSION}

In this paper we proposed TEDDEEC (Threshold Enhanced Developed Distributed Energy Efficient Clustering) protocol which improves stability and energy efficient property of the heterogeneous wireless sensor network and hence increases the lifetime. Simulation results show that TEDDEEC performs better as compared to SEP and DEEC in heterogeneous environment for wireless sensor networks. 


\section{REFERENCES}

[1] Javad Akbari Torkestani, "An adaptive energy-efficient area coverage algorithm for wireless sensor networks," Ad Hoc Networks, vol. 11, pp. 1655-1666, March 2013.

[2] Miloud Bagaa, Yacine Challal, Abdelraouf Ouadjaout, Noureddine Lasla, Nadjib Badachea, Efficient data aggregation with in-network integrity control of WSN, J. Parallel Distrib. Comput., vol. 72, pp. 1157-1170, 21 June 2012.

[3] Li Qing, Qingxin Zhu, Mingwen Wang, "Design of a distributed energy-efficient clustering algorithm for heterogeneous wireless sensor networks," Computer Communications, vol. 29, pp. 2230-2237, 10 March 2006.

[4] O. Younis, S. Fahmy, HEED: "A hybrid, energy-efficient, distributed clustering approach for ad hoc sensor networks", IEEE Transactions on Mobile Computing 3, vol. 4, pp. 660-669, 2004.

[5] S. Lindsey, C.S. Raghavenda, PEGASIS: "power efficient gathering in sensor information systems", in: Proceeding of the IEEE Aerospace Conference, Big Sky, Montana, March 2002.

[6] Georgios Smaragdakis, Ibrahim Matta, Azer Bestavros, "SEP: A Stable Election Protocol for clustered heterogeneous wireless sensor networks", supported in part by NSF grants ITR ANI-0205294, EIA-0202067, ANI0095988, and ANI-9986397.

[7] Li Qing, Qingxin Zhu, Mingwen Wang," Design of a distributed energy-efficient clustering algorithm for heterogeneous wireless sensor networks", Computer Communications, vol. 29, pp. 2230-2237, 2006.

[8] Brahim Elbhiri, Saadane Rachid, Sanaa El fkihi and Driss Aboutajdine, "Developed Distributed Energy-Efficient Clustering (DDEEC) for heterogeneous wireless sensor networks", in proceedings of IEEE, 2010.

[9] Parul Saini and Ajay K. Sharma, "E-DEEC- Enhanced Distributed Energy Efficient Clustering Scheme for heterogeneous WSN", in proceedings of $1^{\text {st }}$ International Conference on Parallel, Distributed and Grid Computing (PDGC), 2010.

[10] N. Javaid,, T. N. Qureshi, A.H. Khan, A. Iqbal, E. Akhtar, M. Ishfaq, "EDDEEC: Enhanced Developed Distributed Energy-Efficient Clustering for HeterogeneousWireless Sensor Networks", Computer Science, vol. 19, pp. 914 - 919 , 2013.

\section{Author(s) Biography}

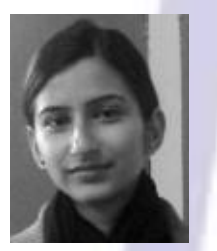

Aditi Sharma received her B.Tech. in Electronics \& Telecommunication from Guru Nanak Dev University, Regional campus Jalandhar, Punjab, India in 2013. She is pursuing her M. Tech. in Electronics \& Communication Engineering from Guru Nanak Dev University, Regional Campus Gurdaspur, Punjab (India). Her research interests is wireless sensor networks. She has published 1 paper in national conference.

Harjit Singh received his B.E. in Electronics \& Telecommunication from Amravati University, Amravati, India in 2002, and the M. Tech. in Electronics \& Communication Engineering from Punjab Technical University, Jalandhar, India, in 2008. He is working as Lecturer in the Department of Electronics and Communication Engineering, Guru Nanak Dev University, Regional Campus, Gurdaspur, Punjab (India). His research interests include digital signal processing, image processing and optical technique. He has published 6 papers in various international/national journals and conferences. He is life member of ISTE and International Association of Engineers (IAENG). 